\title{
Clinical characteristics and prognostic significance of TCGA and ACRG classification in gastric cancer among the Chinese population
}

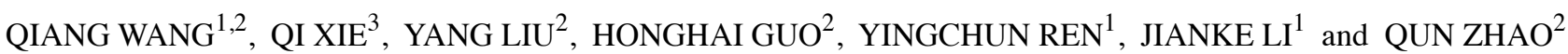 \\ ${ }^{1}$ Department of Thoracic Surgery, The First Hospital of Hebei Medical University, Shijiazhuang, \\ Hebei 050031; ${ }^{2}$ Third Department of General Surgery; ${ }^{3}$ Department of Clinical Nutrition, \\ The Fourth Hospital of Hebei Medical University, Shijiazhuang, Hebei 050011, P.R. China
}

Received June 4, 2019; Accepted March 30, 2020

DOI: $10.3892 / \mathrm{mmr} .2020 .11183$

\begin{abstract}
Molecular classifications of gastric cancer (GC) by the Asian Cancer Research Group (ACRG) and The Cancer Genome Atlas Consortium (TCGA) are useful for diagnosis and treatment of GC. However, their clinical significance is unknown. The present study aims to explore the associations between subtypes of GC and prognosis of patients with GC. Immunohistochemistry (IHC) was used in the ACRG molecular classification of GC, while next-generation sequencing technology was used in TCGA molecular classification. The results indicated that, out of a total of 65 cases of GC, some were classified as Epstein-Barr virus positive type $(9.2 \%, 6$ of 65$)$, some as microsatellite instability (MSI) type $(23.1 \%, 15$ of 65$)$, some as gene stable type $(21.5 \%, 14$ of 65) and some as chromosome instability type (46.2\%, 30 of 65) according to TCGA typing standard. Of the total $65 \mathrm{GC}$ cases, some were classified as MSI (21.5\%, 14 of 65), some as microsatellite stable/epithelial-mesenchymal transition (MSS/EMT; $20.0 \%, 13$ of 65 ), some as MSS/tumor protein 53 active (TP53 $15.4 \%, 10$ of 65$)$ and some as MSS/TP53 inactive (43.1\%, 28 of 65) according to ACRG typing standard. ARCG molecular subtype $(\mathrm{P}=0.010)$ and Lauren classification $(\mathrm{P}=0.011)$ were independently correlated with the overall survival of patients with GC. In conclusion, TCGA classification based on a Chinese population is the same as TCGA typing based on a European population in terms of proportion and clinical characteristics, but there are differences in gene amplification and gene mutation. ACRG molecular classification could be performed by IHC analysis and may be a valuable independent prognostic marker for patients with GC.
\end{abstract}

Correspondence to: Dr Qun Zhao, Third Department of General Surgery, The Fourth Hospital of Hebei Medical University, 14 Jiankang Road, Shijiazhuang, Hebei 050011, P.R. China E-mail: zhaoqun516@126.com

Key words: gastric cancer, Asian Cancer Research Group, The Cancer Genome Atlas Consortium, next-generation sequencing, immunohistochemical staining, prognosis

\section{Introduction}

Gastric cancer (GC) remains the fifth most common type of malignant tumor and is the third leading cause of cancer-related death in the world (1). According to 2015 statistics, nearly 679,100 new cases of GC and 498,000 new mortalities due to GS were diagnosed in China (2). Accurate classification is helpful to the diagnosis, treatment and prognosis of GC. Patient outcome is difficult to predict using only the classic histological criteria and traditional histopathological classification has limited use in guiding clinical outcomes (3). The wide application of next-generation sequencing (NGS) technology has facilitated the molecular classification of numerous tumors (4). It has also helped to define the complex genome landscape of GC more comprehensively and guide its treatment and prognosis $(5,6)$. Therefore, it is particularly important to study the molecular characteristics of GC.

In 2014, on the basis of key DNA defects and molecular abnormalities, The Cancer Genome Atlas Consortium (TCGA) divided GS into Epstein-Barr virus (EBV) positive type, microsatellite instability (MSI) type, gene stable (GS) type and chromosome instability (CIN) type (5). TCGA typing was based on Europe and the USA populations; however, the clinical characteristics of TCGA typing in the Asian population, and its association with clinical parameters and prognosis remain unclear. A new classification method has been proposed by the Asian Cancer Research Group (ACRG) (6). Specifically, four molecular subtypes were proposed: MSI, microsatellite stable (MSS)/epithelial-mesenchymal transition (EMT), MSS/tumor protein 53 (TP53) active and MSS/TP53 inactive. ACRG typing was largely based on Asian populations, mainly from Japan and Korea. However, it is unclear whether ACRG classification standards can be applied to Chinese populations. To reduce the costs, the ACRG recommends the use of immunohistochemistry (IHC) for tumor classification rather than NGS. The MSI type can be identified by MutL protein homolog 1 (MLH1) expression, while the MSS/EMT type can be identified by assay of E-cadherin gene 1 (CDH1) expression and the MSS/TP53 ${ }^{+}$and MSS/TP53 types can be identified by assays of mouse double minute 2 homolog (MDM2) and cyclin-dependent kinase inhibitor 1A (also known as P21) 
expression (6). These four subtypes of GC have different clinical correlations with TCGA subtypes (7). However, based on the Chinese population, the clinical characteristics of TCGA typing and ACRG typing and their predictive role in prognosis remain unclear.

In the present study, NGS technology and IHC staining assays were used for comprehensive analyses of $\mathrm{GC}$, and 65 patients with GC were classified according to different classification criteria. The association between GC molecular classifications, clinicopathological features and prognosis was evaluated. It was further clarified whether TCGA typing and ACRG typing based on IHC methods could be applied to Chinese populations. The present study also evaluated which molecular typing of GC could evaluate prognosis most accurately.

\section{Materials and methods}

Patients and tissue samples. A total of 65 patients with GC (age range, 43-84 years; male: female, 2.10:1) were enrolled at the Third Department of Surgery, The Fourth Hospital of Hebei Medical University, between August 2013 and November 2015. After surgery, all the specimens were divided into sections. One section was sent for clinical pathological diagnosis by pathologists. Without affecting the clinicopathological diagnosis, the tumor tissues visible to the human eye were formalin-fixed at room temperature overnight and paraffin-embedded (FFPE) for routine IHC analysis; $4-\mu \mathrm{m}$ thick paraffin sections were prepared for subsequent experiments. For each patient, only one piece of GC tissue was collected for the experiment. FFPE tissue blocks from primary stomach were available for further experiments. All diagnoses were reviewed by two experienced pathologists and confirmed by hematoxylin for $1 \mathrm{~min}$ and eosin staining for $3 \mathrm{~min}$, both at room temperature. In order to exclude the influence of preoperative adjuvant chemotherapy and radiotherapy on the survival of patients, patients who had not undergone preoperative adjuvant chemotherapy or radiotherapy were selected. However, almost all patients with stage III received chemotherapy after the operation, so patients in the present study were in stage III. Patients with primary malignant tumors in other organs were excluded from the study. Those whose information was incomplete were excluded from the analysis. The clinicopathological data were collected retrospectively from the case history of the patients, including gender, age, Lauren classification, tumor location and postoperative adjuvant therapy. All the patients provided written informed consent before enrollment. The study was approved by the Medical Ethics Committee of The Fourth Hospital of Hebei Medical University.

RNA and DNA extraction, and quantitative determination. Sections $(10-\mu \mathrm{m})$ were cut from the FFPE blocks and each section was transferred into a microcentrifuge tube. Deparaffinization was performed by adding $1 \mathrm{ml}$ xylene for $10 \mathrm{~min}$ twice and $1 \mathrm{ml}$ absolute ethanol for $10 \mathrm{~min}$ twice. RNA and DNA were extracted from PE tissues of GC using High Pure FFPET RNA Isolation kit (Roche Diagnostics GmbH) and DNA FFPE Tissue kit (Qiagen, Inc.). The main steps included dewaxing, dissociation, adsorption and elution. The RNA concentration and purity were routinely measured by
NanoDrop 2000 UV-Vis spectrophotometer (Thermo Fisher Scientific, Inc.), and then RNA was reverse transcribed to cDNA using the RevertAid First Strand cDNA Synthesis kit (Thermo Fisher Scientific, Inc.) at $42^{\circ} \mathrm{C}$ for $60 \mathrm{~min}, 72^{\circ} \mathrm{C}$ for $15 \mathrm{~min}$ and $4^{\circ} \mathrm{C}$ for preservation. The DNA concentration and quality were determined by fluorescence quantitative PCR (qPCR) using the SYBR Green PCR Master mix (Thermo Fisher Scientific, Inc.). The following primer pairs were used for the qPCR: GAPDH forward, 5'-CGCTGAGTACGTCGT GGAGTC-3' and reverse, 5'-GTGATGATCTTGAGGCTG TTGTC-3'. The following thermocycling conditions were used for the qPCR: $94^{\circ} \mathrm{C}$ for $5 \mathrm{~min}$; followed by 35 cycles of $94^{\circ} \mathrm{C}$ for $15 \mathrm{sec}, 58^{\circ} \mathrm{C}$ for $30 \mathrm{sec}$ and $72^{\circ} \mathrm{C}$ for $30 \mathrm{sec}$; and a final extension at $72^{\circ} \mathrm{C}$ for $5 \mathrm{~min}$. Protease digestion of the DNA samples was performed overnight at $55^{\circ} \mathrm{C}$ with gentle rotation. Heat treatment at $95^{\circ} \mathrm{C}$ for 30 min was included or omitted after digestion to validate the heat treatment. All DNA samples were purified by ethanol precipitation and dissolved in distilled water. The DNA concentration and purity were routinely measured by NanoDrop 2000 UV-Vis spectrophotometer (Thermo Fisher Scientific, Inc.). RNA or DNA samples with an optical density (OD)260/OD280 ratio ranging from 1.8 to 2.1 were deemed acceptable.

Library construction and preparation of the sequencing template. The procedure included targeted amplification of the genome region, connection of bar codes to amplified fragments using DNA ligases, purification of magnetic beads from the library and quantitative analysis of the library by fluorescence qPCR. Specimens were diluted to a suitable concentration for the mixed library and the library was then amplified by PCR (Ion One Touch2 System; Thermo Fisher Scientific, Inc.). PCR products were dissociated into single-strand DNA for concentrating positive sequencing template using a template enrichment system (Ion One Touch ES; Thermo Fisher Scientific, Inc.).

Ion Torrent PGM sequencing and analysis. Quality control microspheres (Ion Torrent PGM ${ }^{\mathrm{TM}}$ Sequencing Reagent; Thermo Fisher Scientific, Inc.) were added to the enriched products. Then, sequencing primers were annealed and extended, and finally incubated with PCR (paired end (PE) read 1 sequencing primer, 5'-ACACTCTTTCCCTACACGACGCTCTTCCGATCT-3' and PE read 2 sequencing primer, 5'-CGGTCTCGGCATTCCTGC TGAACCGCTCTTCCGATCT-3'. The above sequencing system was loaded on an Ion Torrent chip (Thermo Fisher Scientific, Inc.), and then the chip was placed on the Ion Torrent $\mathrm{PGM}^{\mathrm{TM}}$ instrument for sequencing. The average sequencing depth was $>2,500 \mathrm{X}$. The original data obtained from sequencing were analyzed by automatic bioinformatics software (ACCB-BIO 2.301; Beijing ACCB Biotech Ltd.), and gene mutations were screened and annotated (mutation abundance threshold, 1\%; positive reads number, $>5$ ). The results of gene variation analysis by ACCB-BIO 2.301 were confirmed by a comprehensive genomics viewer (Integrative Genomics Viewer; Broad Institute; http://software.broadinstitute.org/software/igv), and clinically analyzable results were generated (8).

IHC staining of the FFPE tissue blocks. The protein expression of MLH1, MDM2, P21, E-cadherin and vimentin was detected 
Table I. The Cancer Genome Atlas molecular subtypes by sequencing.

\begin{tabular}{|c|c|c|}
\hline Molecular subtype & Index & Positive result \\
\hline \multirow[t]{6}{*}{$\mathrm{EBV}+$} & EBV positive & 6 \\
\hline & PLK3CA mutation & 0 \\
\hline & Hypermethylation & 0 \\
\hline & $J A K 2$ amplification & 0 \\
\hline & $C D 274(P D-L 1)$ amplification & 0 \\
\hline & PDCD1LG2 (PD-L2) amplification & 0 \\
\hline \multirow[t]{3}{*}{ MSI } & MSI status & 15 \\
\hline & Hypermutated & 0 \\
\hline & Hypermethylation & 0 \\
\hline \multirow[t]{4}{*}{ GS } & RHOA mutation & 0 \\
\hline & CLDN18-ARHGAP26 rearrangement & 0 \\
\hline & CLDN18-ARHGAP6 rearrangement & 0 \\
\hline & Absence of extensive somatic copy-number aberrations & 14 \\
\hline \multirow[t]{2}{*}{ CIN } & Copy Number Cluster (Aneuploidy) & 30 \\
\hline & $\begin{array}{l}\text { Focal amplification of receptor tyrosine kinases (vascular endothelial } \\
\text { growth factor receptor 2) }\end{array}$ & 0 \\
\hline
\end{tabular}

EBV, Epstein-Barr virus; MSI, microsatellite instability; GS, gene stable; CIN, chromosome instability.

with a streptavidin-biotin peroxidase kit (SP-9001/9002; OriGene Technologies, Inc.), according to the manufacturer's protocol. Briefly, non-specific sites were blocked with normal sheep serum (Reagent A from the kit) for $1 \mathrm{~h}$ at $37^{\circ} \mathrm{C}$. MLH1 (1:50; clone EPR3894; cat. no. ab92312; Abcam), MDM2 (1:100; clone SMP14; cat. no. sc-965; Santa Cruz Biotechnology, Inc.), P21 (1:100; clone EPR362; cat. no. ab109520; Abcam), E-cadherin (1:100; clone 5F133; cat. no. sc-71007; Santa Cruz Biotechnology, Inc.) and vimentin (1:50; clone V9; cat. no. sc-6260; Santa Cruz Biotechnology, Inc.) rabbit/mouse anti-human antibodies were incubated with the sections at $4^{\circ} \mathrm{C}$ overnight. Biotinylated goat anti-rabbit/mouse antibody (Reagent B from the kit) and horseradish peroxidase-labeled streptase ovalbumin (3rd antibody; Reagent $\mathrm{C}$ from the kit) were incubated with the sections at $37^{\circ} \mathrm{C}$ for $30 \mathrm{~min}$ each. Following staining with diaminobenzidine reagent (ZLI-9018; OriGene Technologies, Inc.), the slices were counterstained with hematoxylin for $2 \mathrm{~min}$ at room temperature and sealed with neutral gum.

Scoring criteria and molecular classification. The final results were independently observed and graded by two pathologists double blinded to the experiment. A total of 5 fields of vision were randomly selected from each section and 100 cells were counted in each field under high-power light microscopy observation (magnification, x200). IHC staining intensity and proportion of positive cells were evaluated by the scoring method of Sinicrope et al (9). The scores of positive cells were assessed as follows: $\leq 5 \%$ stained cells, $0 ; 6-25 \%$ stained cells, $1 ; 26-50 \%$ stained cells, $2 ; 51-75 \%$ stained cells, 3 ; and $75 \%$ stained cells, 4 . The scores of intensity were as follows: Negative staining, 0; weak staining, 1; moderate staining, 2; and strong staining, 3 (Fig. S1). By multiplying the scores of positive cells and intensity, the final score of each tumor specimen was obtained.

For MLH1 expression, a final score $\geq 1$ was defined as positive expression $(10,11)$. For MDM2, p21, E-cadherin and vimentin expression, a final score of $0-2$ was defined as negative expression, while 3-12 was defined as positive expression (5). According to the ACRG molecular classification, GC cases with MLH1 negative expression were categorized as MSI, while GC cases with MLH1 positive expression were categorized as MSS. Among the MSS cases, GC with E-cadherin (-) and vimentin $(+)$ were categorized as MSS/EMT, while those with MDM2 (-) and P21 (+) were categorized as MSS/TP53+. GC cases with MDM2 (+) and P21 (-) were categorized as MSS/TP53- (6). According to TCGA molecular classification, EBV-positive $\mathrm{GC}$ cases were classified as the $\mathrm{EBV}^{+}$subtype. Among the EBV-negative specimens, MSI-high stable GC was categorized as MSI. The other specimens were categorized as GS or CIN subtype according to their degree of aneuploidy (5).

Statistical analysis. SPSS 23.0 software (IBM Corp.) was used for all the statistical analyses. Frequency data were analyzed by $\chi^{2}$ or Fisher's exact test for categorical variables, as appropriate. Overall survival (OS) was calculated from the time of diagnosis to the time of death from any cause or to the last follow-up date. The Kaplan-Meier method, log-rank test and Cox's proportional hazards regression model were used for univariate survival analysis. The logistic regression model was used to analyze the single factors, while multivariate analysis was used during stepwise regression for statistically significant variables in single factor analysis. Multivariate survival analysis was performed by the Cox's proportional hazards regression model. Heat maps were used to assess the overall mutation pattern, amplification pattern and the patterns 
Table II. The Cancer Genome Atlas molecular subtypes and characteristics of patients with GC.

\begin{tabular}{|c|c|c|c|c|c|}
\hline Clinicopathological factors & $\mathrm{EBV}^{+}, \mathrm{n}(\%)$ & MSI, n (\%) & $\mathrm{GS}, \mathrm{n}(\%)$ & CIN, n (\%) & P-value \\
\hline Sex & & & & & 0.238 \\
\hline Male & $5(7.7)$ & $7(10.8)$ & $10(15.4)$ & $22(33.8)$ & \\
\hline Female & $1(1.5)$ & $8(12.3)$ & $4(6.2)$ & $8(12.3)$ & \\
\hline Age/year & & & & & $<0.001$ \\
\hline$\leq 60$ & $3(4.6)$ & $1(1.5)$ & $12(18.5)$ & $18(27.7)$ & \\
\hline$>60$ & $3(4.6)$ & $14(21.5)$ & $2(3.1)$ & $12(18.5)$ & \\
\hline Tumor location & & & & & 0.008 \\
\hline EGJ & $2(3.1)$ & $3(4.6)$ & $7(10.8)$ & $17(26.2)$ & \\
\hline Distal stomach & $4(6.2)$ & $12(18.5)$ & $7(10.8)$ & $13(20.0)$ & \\
\hline Lauren classification & & & & & 0.582 \\
\hline Diffuse & $2(3.1)$ & $3(4.6)$ & $5(7.7)$ & $8(12.3)$ & \\
\hline Intestinal & $2(3.1)$ & $5(7.8)$ & $6(9.2)$ & $16(24.6)$ & \\
\hline Mixed & $2(3.1)$ & $7(10.8)$ & $3(4.6)$ & $6(9.2)$ & \\
\hline Adjuvant therapy & & & & & 0.833 \\
\hline XELOX & $3(4.6)$ & $7(10.8)$ & $6(9.2)$ & $17(26.2)$ & \\
\hline SOX & $3(4.6)$ & $8(12.3)$ & $8(12.3)$ & $13(20.0)$ & \\
\hline
\end{tabular}

GC, gastric cancer; EBV, Epstein-Barr virus; MSI, microsatellite instability; GS, gene stable; CIN, chromosome instability; EGJ, gastro-esophageal junction; XELOX, capecitabine combined with oxaliplatin; SOX, S1 combined with oxaliplatin.

A TCGA gastric tumors

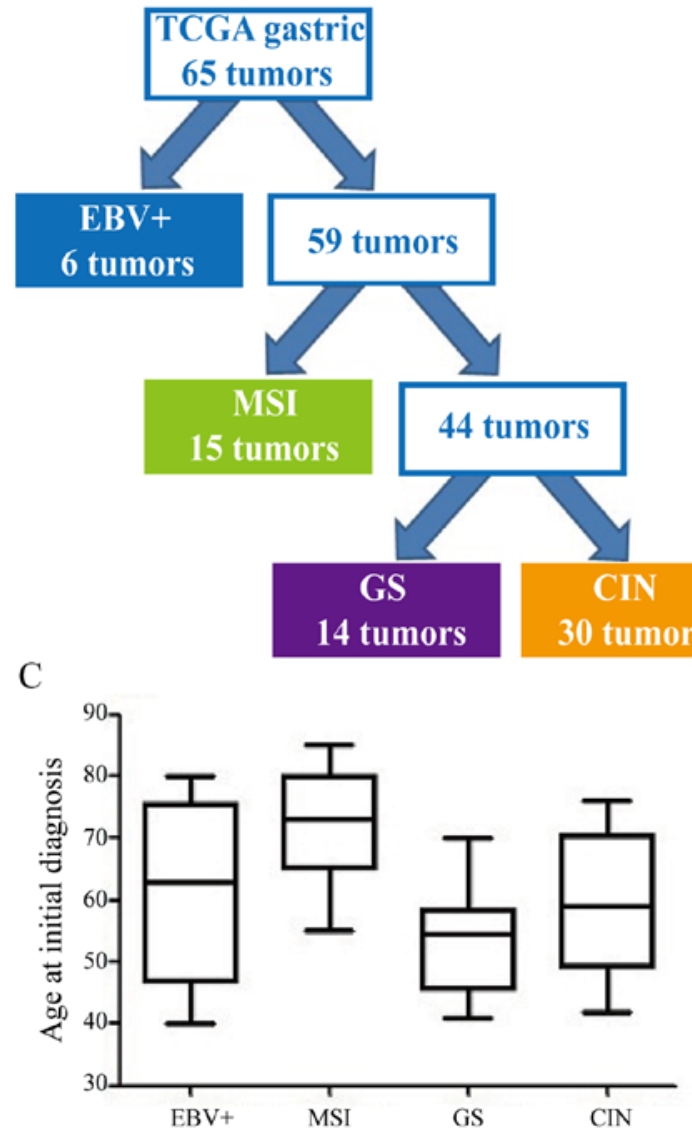

B

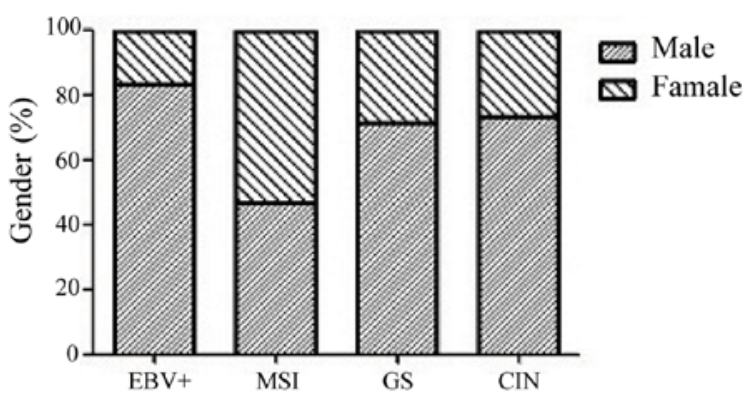

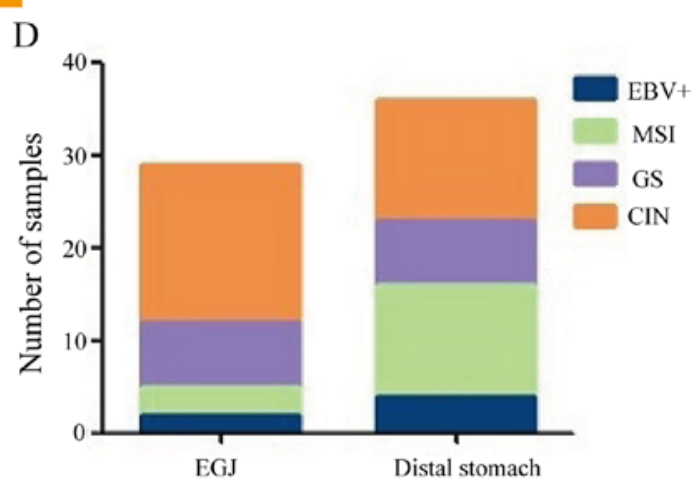

Figure 1. TCGA molecular classification of GC. (A) Flow diagram illustrating how GC divided according to TCGA molecular classification. (B) Differences in gender among subtypes. (C) Differences in age among classification. (D) Differences in tumor location among classification. TCGA, The Cancer Genome Atlas; GC, gastric cancer; EBV, Epstein-Barr virus; MSI, microsatellite instability; GS, gene stable; CIN, chromosome instability; EGJ, gastro-esophageal junction. 

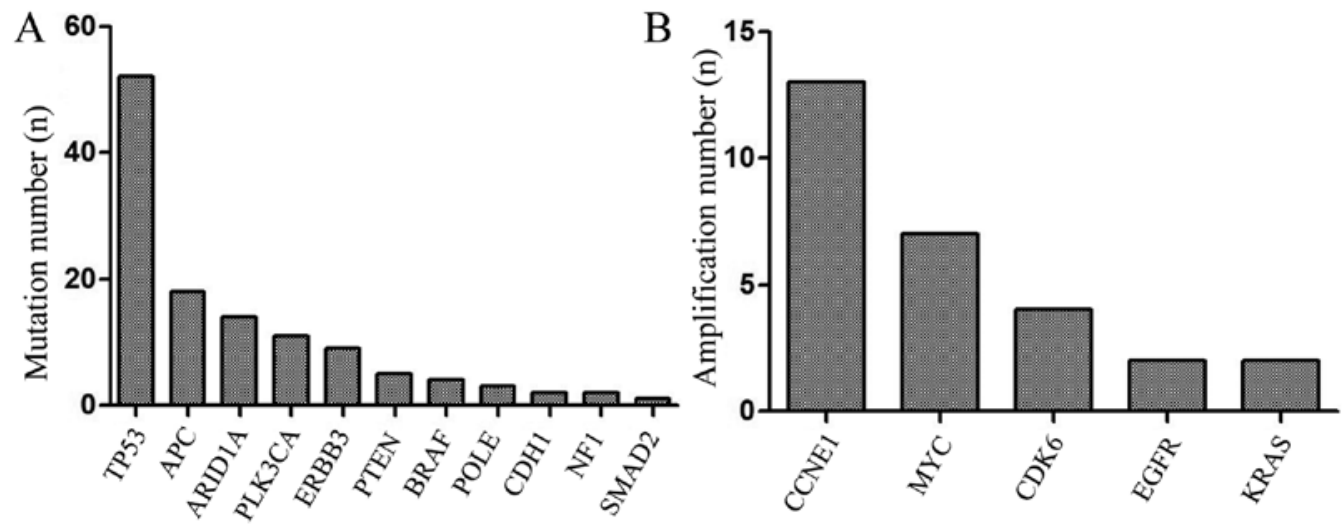

Figure 2. Significantly mutated and amplified genes in gastric cancer. (A) Mutated genes. (B) Amplified genes.

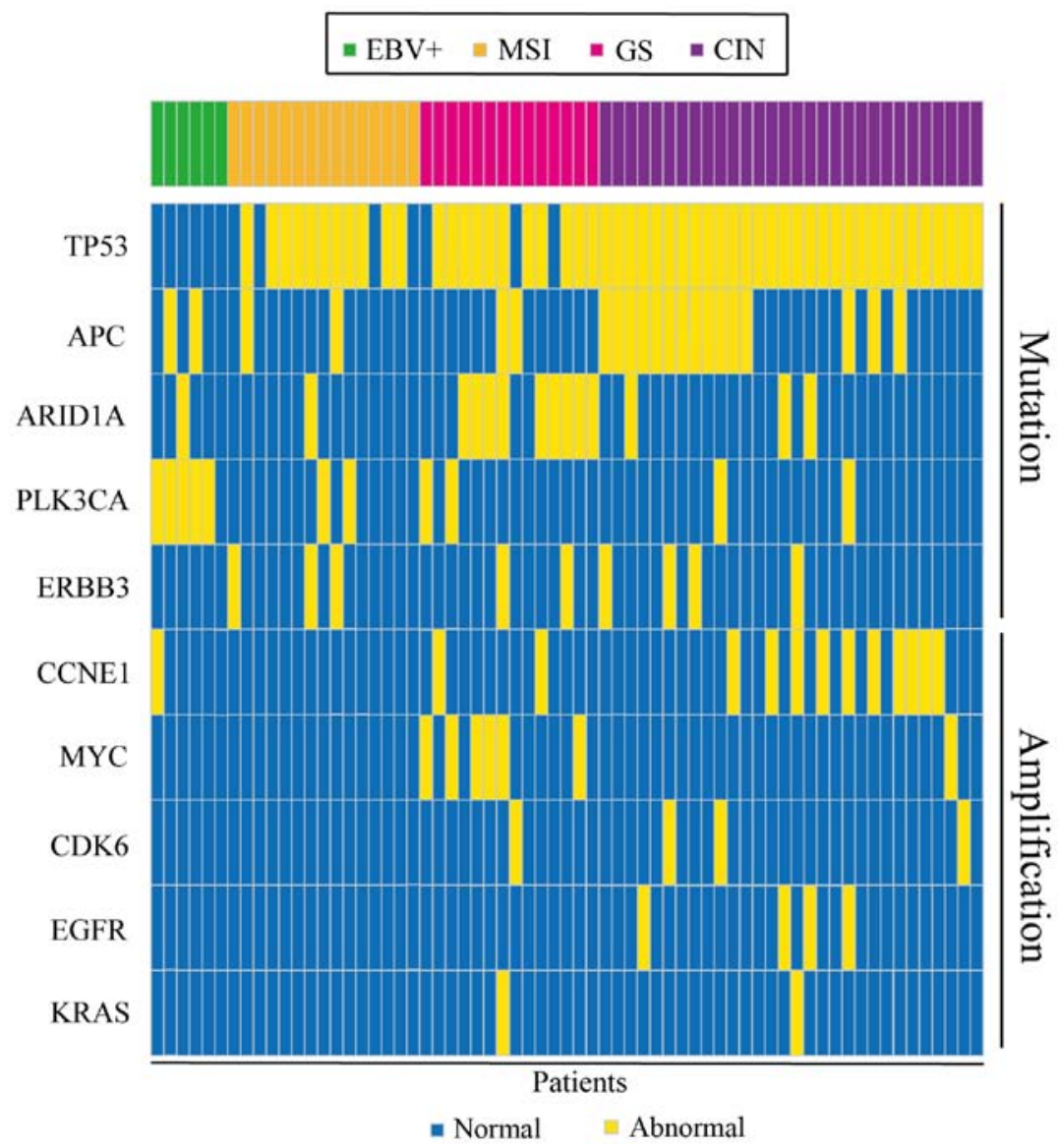

Figure 3. Heat map for the mutated and amplified genes. EBV, Epstein-Barr virus; MSI, microsatellite instability; GS, gene stable; CIN, chromosome instability.

by type. All statistical tests were two-sided and $\mathrm{P}<0.05$ was considered to indicate as statistically significant difference.

\section{Results}

Patient baseline characteristics. A cohort of 65 patients, comprising 44 males $(67.7 \%)$ and 21 females (32.3\%), with a median of 62 years of age and a range of 45-80 years of age, were included. All patients enrolled in the study received surgical treatment and were pathologically diagnosed with GC carcinoma. In total, $44.62 \%(29 / 65)$ of the tumors were located at the gastroesophageal junction and $55.38 \%(36 / 65)$ of the tumors were located at the distal stomach. None of the patients received preoperative adjuvant radiotherapy or neoadjuvant therapy; 33 patients received capecitabine combined with oxaliplatin (XELOX) postoperative adjuvant chemotherapy and 32 patients received S1 combined with oxaliplatin postoperative adjuvant chemotherapy. There were no perioperative mortalities. According to the eighth edition of the AJCC Cancer Staging Manual, all patients were in stage III and 18 (27.7\%) patients were diagnosed with diffuse Lauren classification tumors, $29(44.6 \%)$ patients were diagnosed with intestinal Lauren classification tumors and 18 (27.7\%) patients were diagnosed with mixed Lauren classification tumors. 

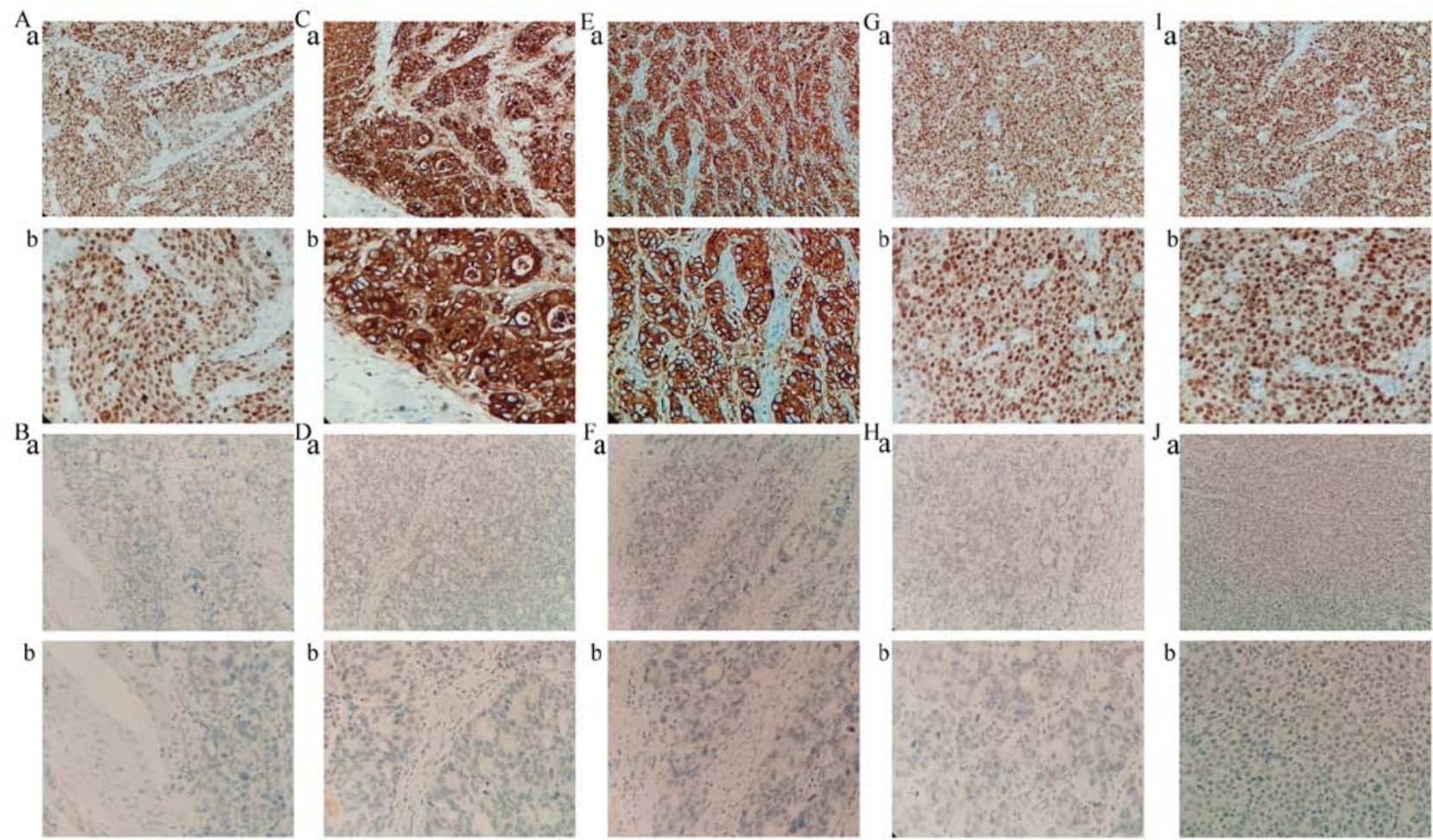

Figure 4. Multiple markers expression in gastric cancer by immunohistochemistry staining. (A) MLH1 positive staining. (B) MLH1 negative staining. (C) E-cadherin positive staining, (D) E-cadherin negative staining. (E) Vimentin positive staining. (F) Vimentin negative staining. (G) MDM2 positive staining. (H) MDM2 negative staining. (I) P21 positive staining and (J) P21 negative staining. Magnification (a), x200; (b), x400. MLH1, MutL protein homolog 1; MDM2, mouse double minute 2 homolog; P21, cyclin-dependent kinase inhibitor 1A.

TCGA molecular subtypes of $G C$ and their association with clinicopathological features. The results of 65 cases of GC according to TCGA classification criteria are shown in Table I. Of the $65 \mathrm{GC}$ cases, 6 (9.2\%) were $\mathrm{EBV}^{+}$subtype, 15 (23.1\%) were MSI subtype, 14 (21.5\%) were GS subtype and 30 (46.2\%) were CIN subtype (Fig. 1A). Table II shows TCGA molecular subtypes and their clinicopathological factors. The MSI subtype was more common in females (53.3\%), while the $\mathrm{EBV}^{+}$subtype was more common in males $(83.3 \%, \mathrm{P}=0.021$; Fig. 1B). The GS subtype was diagnosed at relatively younger ages (median age, 54 years), whereas the MSI subtype was diagnosed at relatively older ages (median age, 72 years; Fig. 1C). The CIN subtype tumors were more common in the gastroesophageal junction $(58.6 \%, \mathrm{P}=0.008$; Fig. 1D). There were no significant differences in gender, Lauren classification or which postoperative adjuvant therapy the patients with GC received in four TCGA subtypes ( $\mathrm{P}>0.05$; Table II).

NGS revealed that the mutation rate of TP53 was $80.0 \%$ (52/65), APC 27.7\% (18/65), AT-rich interactive domain 1A (ARIDIA) $21.5 \%$ (14/65), of PLK3CA $16.9 \%(11 / 65)$, of ERBB3 13.8\% (9/65), of PTEN 7.7\% (5/65), of BRAF 6.2\% (4/65), of POLE 4.6\% (3/65), of CDH1 3.1\% (2/65), of NF1 3.1\% (2/65) of SMAD2 1.5\% (1/65; Fig. 2A). TP53 and $P L K 3 C A$ are the most frequently mutated genes in human tumors, and are associated with poor prognosis in various cancer types, since they play a vital role in tumor immune regulation. Mutations in $A P C$ would lead to alterations in cell signal transduction, differentiation, mediation of intercellular adhesion, stabilization of the cytoskeleton, and regulation of the cell cycle and apoptosis. ARIDIA mutations could increase immune activity in gastrointestinal cancer. The results of the present study also revealed that the amplification percentage of CCNE1 was $20.0 \%$ (13/65), of MYC $10.8 \%$ (7/65), of CDK6 6.2\% (4/65), of EGFR $3.1 \%$ (2/65) and of KRAS 1.5\% (2/65) (Fig. 2B). Gene mutations and gene amplification are shown in Fig. 3. The distribution of mutated genes and amplified genes in gastric cancer typing is shown in Table SI: As demonstrated in Fig. 3, TP53 mutations were mostly identified in patients with CIN, with a mutation percentage of $57.7 \%$ (Table SI), and similar results were obtained for APC (66.7\%; Table SI) and ERBB3 (44.4\%; Table SI). ARIDIA mutations were mainly identified in the GS type (64.3\%; Table SI) and PLK3CA mutations in the $\mathrm{EBV}^{+}$type (45.5\%; Table SI). In addition, $C C N E 1$ amplification was predominantly found in patients with CIN (76.9\%; Fig. 3; Table SI), with similar results obtained for CDK6 and EGFR (75 and 100\% respectively; Table SI). Conversely, MYC amplifications were predominantly identified in the GS type (85.7\%; Table SI).

ACRG molecular subtypes of GC and their association with clinicopathological features. IHC staining revealed that MLH1, MDM2 and P21 proteins were mainly located in the nucleus, whereas E-cadherin and vimentin proteins were mainly expressed in the cellular cytoplasm and membrane of tumor cells (Fig. 4). The expression of MLH1, MDM2, P21, E-cadherin and vimentin, as well as the clinicopathological characteristics of patients, are shown in Table III. MLH1 and MDM2 proteins were significantly associated with age $(\mathrm{P}<0.05)$. E-cadherin and vimentin proteins were 


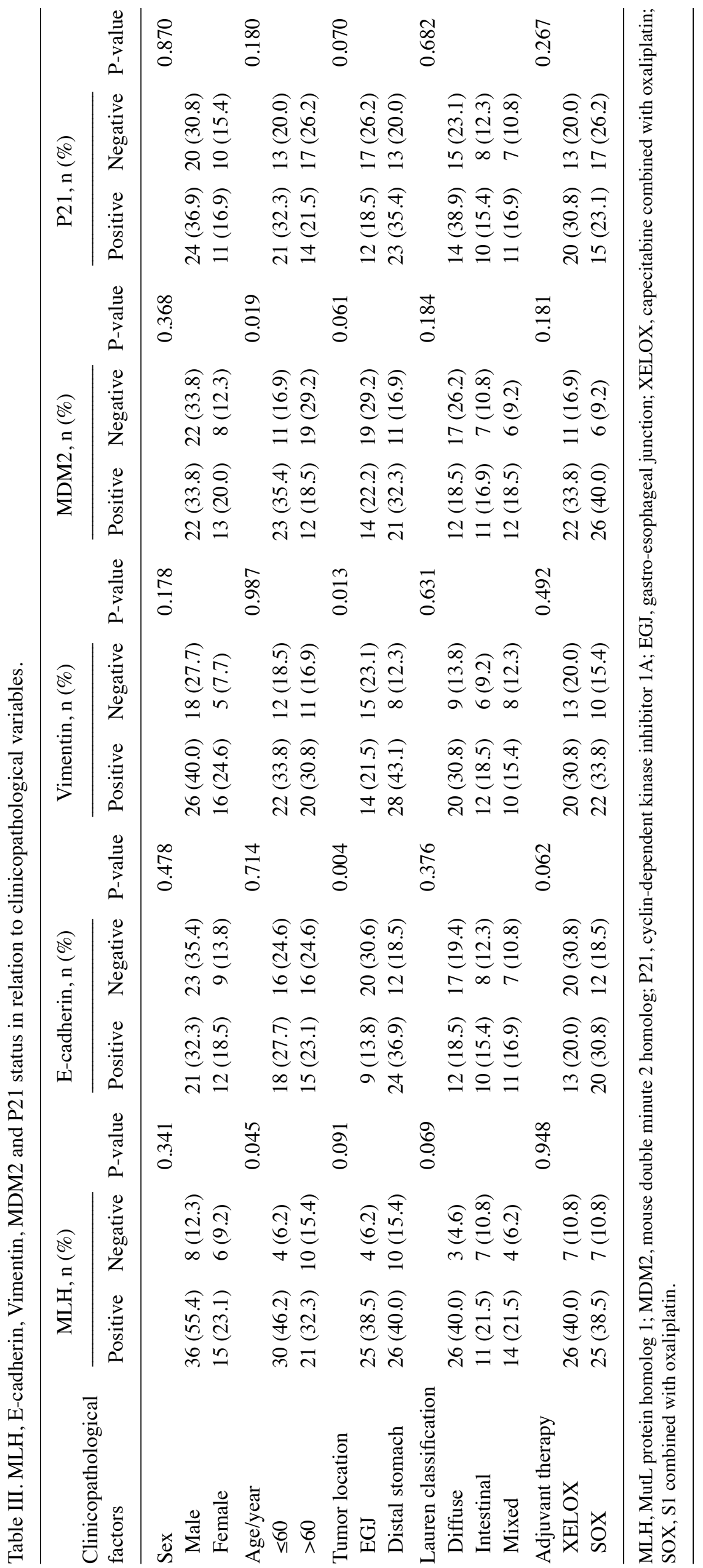


Table IV. Asian Cancer Research Group molecular subtypes and characteristics of gastric cancer patients.

\begin{tabular}{|c|c|c|c|c|c|}
\hline Clinicopathological factors & MSI, n (\%) & MSS/EMT, n (\%) & MSS/TP53+, n (\%) & MSS/TP53 $3^{-}$n (\%) & P-value \\
\hline Sex & & & & & 0.818 \\
\hline Male & $8(12.3)$ & $9(13.8)$ & $7(10.8)$ & $20(30.8)$ & \\
\hline Female & $6(9.2)$ & $4(6.2)$ & $3(4.6)$ & $8(12.3)$ & \\
\hline Age/year & & & & & 0.066 \\
\hline$\leq 60$ & $4(6.2)$ & $10(15.4)$ & $4(6.2)$ & $16(24.6)$ & \\
\hline$>60$ & $10(15.4)$ & $3(4.6)$ & $6(9.2)$ & $12(18.5)$ & \\
\hline Tumor location & & & & & 0.136 \\
\hline EGJ & $4(6.2)$ & $9(13.8)$ & $3(4.6)$ & $13(20.0)$ & \\
\hline Distal stomach & $10(15.4)$ & $4(6.2)$ & $7(10.8)$ & $15(23.1)$ & \\
\hline Lauren classification & & & & & 0.125 \\
\hline Diffuse & $3(4.6)$ & $7(10.8)$ & $3(4.6)$ & $5(7.7)$ & \\
\hline Intestinal & $7(10.8)$ & $4(6.2)$ & $2(3.1)$ & $16(24.6)$ & \\
\hline Mixed & $4(6.2)$ & $2(3.1)$ & $5(7.7)$ & $7(10.8)$ & \\
\hline Adjuvant therapy & & & & & 0.996 \\
\hline XELOX & $7(10.8)$ & $7(10.8)$ & $5(7.7)$ & $14(21.5)$ & \\
\hline SOX & $7(10.8)$ & $6(9.2)$ & $5(7.7)$ & $14(21.5)$ & \\
\hline
\end{tabular}

MSI, microsatellite instability; MSS, microsatellite stable; EMT, epithelial-mesenchymal transition; TP53, tumor protein 53; EGJ, gastro-esophageal junction; XELOX, capecitabine combined with oxaliplatin; SOX, S1 combined with oxaliplatin.
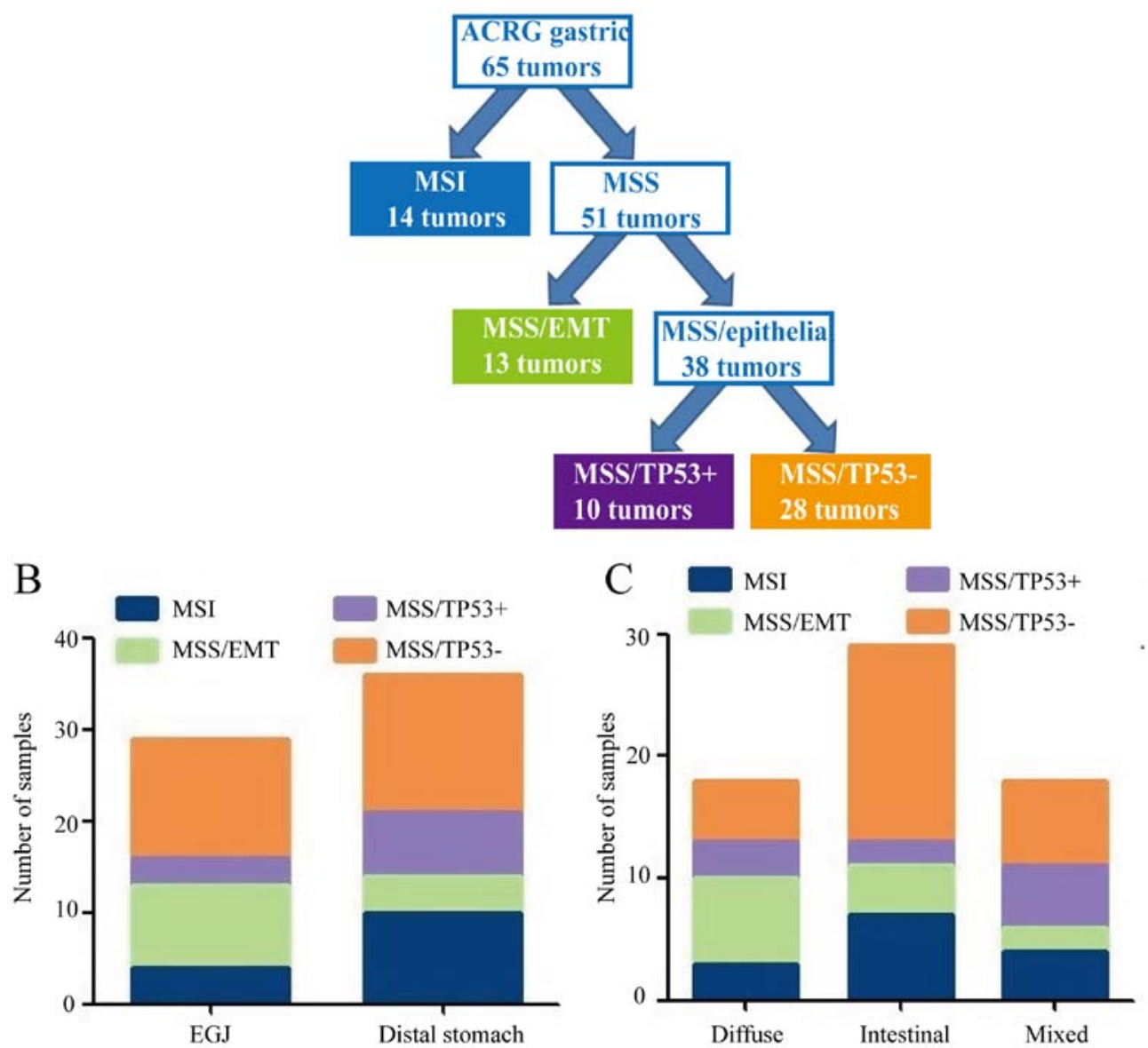

Figure 5. ACRG molecular classification of GC. (A) Flow diagram illustrating how GC divided according to ACRG molecular classification. (B) Differences in tumor location among subtypes. (C) Differences in Lauren classification among subtypes. ACRG, Asian Cancer Research Group; GC, gastric cancer; MSI, microsatellite instability; MSS, microsatellite stable; EMT, epithelial-mesenchymal transition; TP53, tumor protein 53; EGJ, gastro-esophageal junction. 

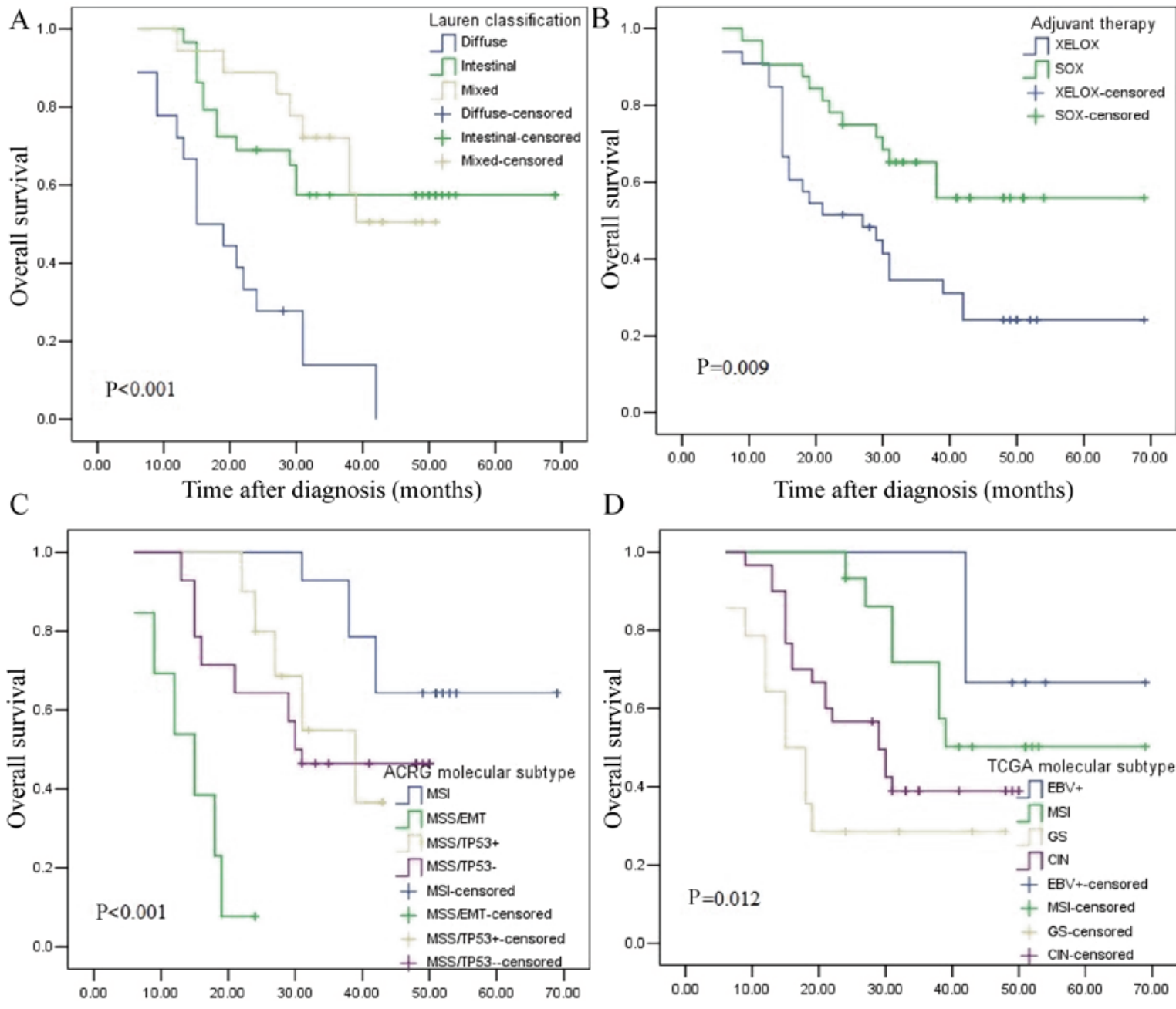

$\mathrm{E}$

Time after diagnosis (months)

$\mathrm{F}$
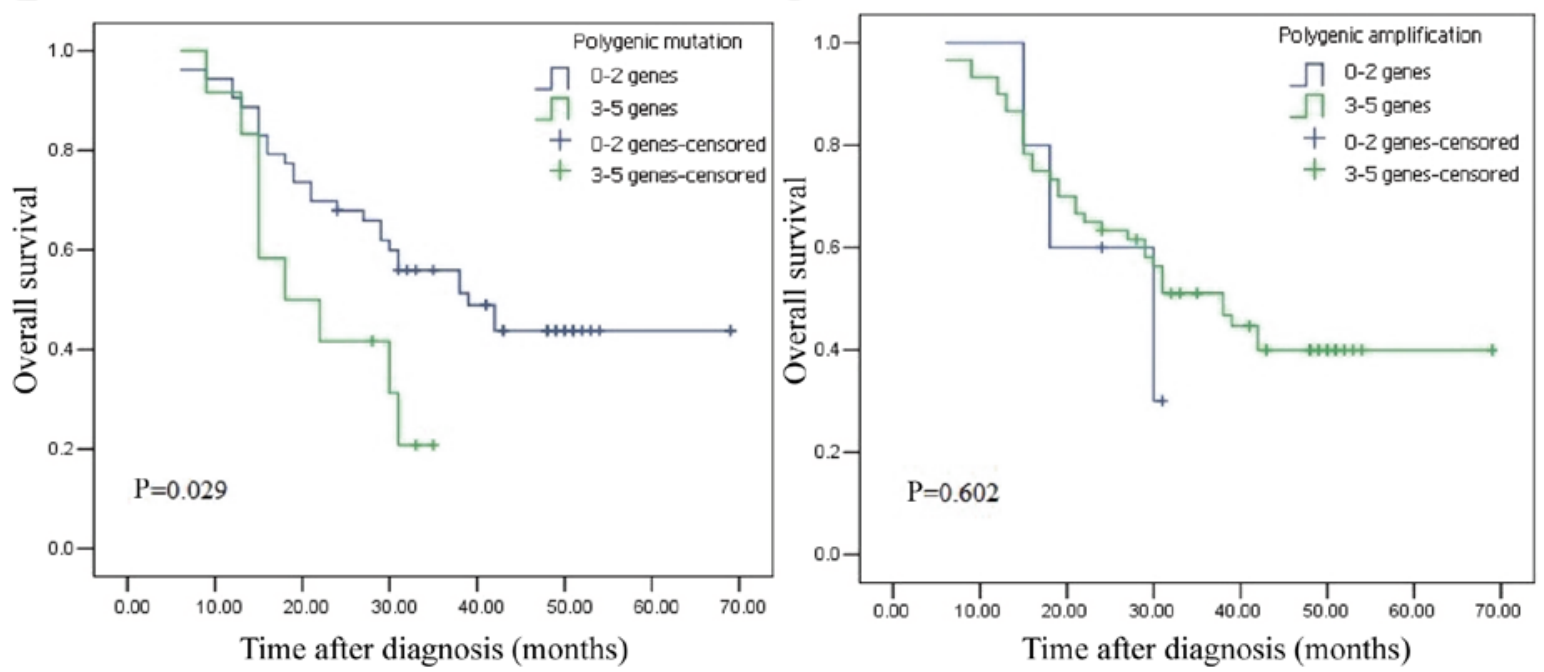

Figure 6. Kaplan-Meier curves for the overall survival of 65 patients with gastric cancer. (A) Lauren classification, (B) postoperative adjuvant chemotherapy, (C) ACRG molecular subtypes, (D) TCGA molecular subtypes, (E) Polygenic mutation and (F) Polygenic amplification. XELOX, capecitabine combined with oxaliplatin; SOX, S1 combined with oxaliplatin; MSI, microsatellite instability; MSS, microsatellite stable; EMT, epithelial-mesenchymal transition; TP53, tumor protein 53; EBV, Epstein-Barr virus; MSI, microsatellite instability; GS, gene stable; CIN, chromosome instability.

significantly associated with tumor location $(\mathrm{P}<0.05)$. Table IV shows the ACRG molecular subtypes and their clinicopathological features. Among the $65 \mathrm{GC}$ cases, 14 (21.5\%) were MSI subtype, 13 (20.0\%) were MSS/EMT subtype, $10(15.4 \%)$ were $\mathrm{MSS} / \mathrm{TP}^{+} 3^{+}$subtype and $28(43.1 \%)$ were
MSS/TP53- subtype (Fig. 5A). MSS/TP53+ tumors showed low frequency in gastro-esophageal junction (EGJ) (10.3\%), whereas the majority of MSS/TP53 tumors were present in the distal stomach (41.7\%) (Fig. 5B). Patients with MSS/EMT GC tended to be of diffuse type (53.8\%), while patients with 
Table V. Univariate analysis and multivariate analysis of prognostic factors in gastric cancer for overall survival.

\begin{tabular}{|c|c|c|c|c|c|c|}
\hline \multirow[b]{2}{*}{ Variable } & \multicolumn{3}{|c|}{ Univariate analysis } & \multicolumn{3}{|c|}{ Multivariate analysis } \\
\hline & HR & P-value & $95 \% \mathrm{CI}$ & HR & P-value & $95 \% \mathrm{CI}$ \\
\hline $\begin{array}{l}\text { TCGA molecular subtype } \\
\text { EBV }^{+} \text {vs. MSI vs. GS vs. CIN }\end{array}$ & 1.070 & 0.600 & $0.832-1.376$ & & & \\
\hline $\begin{array}{l}\text { ACRG molecular subtype } \\
\text { MSI vs. MSS/EMT vs. MSS/TP53+ vs. MSS/TP53- }\end{array}$ & 1.381 & 0.045 & $1.007-1.894$ & 1.514 & 0.010 & $1.106-2.073$ \\
\hline $\begin{array}{l}\text { Sex } \\
\text { Male vs. Female }\end{array}$ & 1.267 & 0.512 & $0.626-2.564$ & & & \\
\hline $\begin{array}{l}\text { Age (years) } \\
\leq 60 \text { vs. }>60\end{array}$ & 1.142 & 0.688 & $0.598-2.179$ & & & \\
\hline $\begin{array}{l}\text { Tumor location } \\
\text { EGJ vs. Distal stomach }\end{array}$ & 1.417 & 0.290 & $0.743-2.705$ & & & \\
\hline $\begin{array}{l}\text { Lauren classification } \\
\text { Intestinal vs. Diffuse vs. Mixed }\end{array}$ & 4.424 & 0.001 & $1.884-10.388$ & 3.321 & 0.011 & $1.311-8.414$ \\
\hline $\begin{array}{l}\text { Adjuvant therapy } \\
\text { XELOX vs. SOX }\end{array}$ & 2.373 & 0.013 & $1.204-4.676$ & 2.002 & 0.068 & $0.950-4.219$ \\
\hline
\end{tabular}

Table VI. Univariate analysis and multivariate analysis of mutant genes in gastric cancer for overall survival.

\begin{tabular}{|c|c|c|c|c|c|c|}
\hline \multirow[b]{2}{*}{ Variable } & \multicolumn{3}{|c|}{ Univariate analysis } & \multicolumn{3}{|c|}{ Multivariate analysis } \\
\hline & HR & P-value & $95 \% \mathrm{CI}$ & HR & P-value & $95 \% \mathrm{CI}$ \\
\hline $\begin{array}{l}\text { TP53 } \\
\text { Normal vs. abnormal }\end{array}$ & 4.797 & 0.010 & $1.465-15.707$ & 4.193 & 0.019 & $1.260-13.945$ \\
\hline $\begin{array}{l}A P C \\
\text { Normal vs. abnormal }\end{array}$ & 1.180 & 0.655 & $0.571-2440$ & & & \\
\hline $\begin{array}{l}\text { ARIDIA } \\
\text { Normal vs. abnormal }\end{array}$ & 1.516 & 0.262 & $0.733-3.135$ & & & \\
\hline $\begin{array}{l}P L K 3 C A \\
\text { Normal vs. abnormal }\end{array}$ & 2.768 & 0.010 & $1.274-6.013$ & 2.114 & 0.061 & $0.966-4.626$ \\
\hline $\begin{array}{l}E R B B 3 \\
\text { Normal vs. abnormal }\end{array}$ & 1.269 & 0.596 & $0.526-3.060$ & & & \\
\hline
\end{tabular}

HR, hazard ratio; CI, confidence interval.

MSS/TP53- GC were of intestinal type (57.1\%; Fig. 5C). There were no obvious differences in gender, age, tumor location, Lauren classification or type of postoperative adjuvant therapy received by patients with $\mathrm{GC}$ in the four TCGA subtypes (P>0.05; Table IV).

TCGA and ACRG molecular subtypes of GC are associated with $O S$. The median follow-up time was 31 months (range, 6-69 months) and the median survival time was 31 months.
Kaplan-Meier plots revealed that the patients with diffuse type, GS molecular subtype, MSS/EMT subtype and those who accepted XELOX adjuvant chemotherapy had the worst outcome (Fig. 6A-D). The OS of four ACRG subtypes was further analyzed. MSI subtype vs. MSS/EMT subtype demonstrated an obviously different prognosis $(\mathrm{P}<0.001)$, as did MSS/EMT subtype vs. MSS/TP53- subtype $(\mathrm{P}<0.001)$. The MSS/EMT subtype had the worst prognosis, followed by MSS/TP53-, MSS/TP53+ and MSI. The EBV ${ }^{+}$subtype 
Table VII. Univariate analysis and multivariate analysis of amplified genes in gastric cancer for overall survival.

\begin{tabular}{|c|c|c|c|c|c|c|}
\hline \multirow[b]{2}{*}{ Variable } & \multicolumn{3}{|c|}{ Univariate analysis } & \multicolumn{3}{|c|}{ Multivariate analysis } \\
\hline & HR & P-value & $95 \% \mathrm{CI}$ & HR & P-value & $95 \% \mathrm{CI}$ \\
\hline $\begin{array}{l}\text { CCNE1 } \\
\text { Normal vs. abnormal }\end{array}$ & 2.190 & 0.035 & $1.058-4.535$ & 1.891 & 0.101 & $0.883-4.054$ \\
\hline $\begin{array}{l}\text { MYC } \\
\text { Normal vs. abnormal }\end{array}$ & 1.468 & 0.472 & $0.515-4.186$ & & & \\
\hline $\begin{array}{l}\text { CDK6 } \\
\text { Normal vs. abnormal }\end{array}$ & 3.996 & 0.013 & $1.333-11.983$ & & & \\
\hline $\begin{array}{l}E G F R \\
\text { Normal vs. abnormal }\end{array}$ & 21.235 & 0.475 & $0.005-92105.54$ & 3.071 & 0.054 & $0.983-9.593$ \\
\hline $\begin{array}{l}\text { KRAS } \\
\text { Normal vs. abnormal }\end{array}$ & 2.279 & 0.260 & $0.544-9.550$ & & & \\
\hline
\end{tabular}

HR, hazard ratio; CI, confidence interval.

exhibited an obvious different prognosis vs. the GS subtype $(\mathrm{P}=0.032)$, while there was no obvious difference in prognosis between the $\mathrm{EBV}^{+}$and $\mathrm{CIN}$ subtypes $(\mathrm{P}=0.088)$. The $\mathrm{EBV}^{+}$ subtype had the best prognosis, followed by MSI, CIN and GS. Univariate analysis revealed that only ARCG molecular subtype $(\mathrm{P}=0.045)$, Lauren classification $(\mathrm{P}=0.001)$ and adjuvant therapy $(\mathrm{P}=0.013)$ were associated with the OS of GC. These three indicators were considered in the regression model for multivariate analysis, which revealed that ARCG molecular subtype $(\mathrm{P}=0.010)$ and Lauren classification $(\mathrm{P}=0.011)$ were associated with the OS of GC (Table V). These results revealed that Lauren classification and ARCG subtype are independent prognostic predictors for GC.

The association between abnormal genes and prognosis was further analyzed. Kaplan-Meier plots revealed that patients with polygenic mutation had a worse outcome (Fig. 6E). Univariate and multivariate analyses (Tables VI and VII) revealed that patients with TP53 mutation (hazard ratio $=4.193,95 \%$ confidence interval $=1.260-13.945$ ) had a poor survival time. These results indicated that TP53 mutation is an independent prognostic predictor for GC.

\section{Discussion}

The present study used NGS technology and IHC staining for comprehensive analyses of GC and classified them according to different classification criteria. It also evaluated the association between GC molecular classifications, clinicopathological features and prognosis. The results revealed that Lauren classification, adjuvant therapy, ARCG molecular subtype, TCGA molecular subtype and polygenic mutation were associated with poor prognosis of patients with GC. Furthermore, it was observed that ACRG classification of GC could evaluate prognosis more accurately than TCGA classification.

In total, 6 cases $(9.2 \%)$ were $\mathrm{EBV}^{+}$type out of $65 \mathrm{GC}$ specimens and patients with $\mathrm{EBV}^{+}$type had the best overall prognosis in the present study. This is consistent with previous studies reporting that the $\mathrm{EBV}^{+}$type of $\mathrm{GC}$ accounted for $~ 9 \%$ (5). A previous study suggested that, in contrast to $\mathrm{EBV}^{-} \mathrm{GC}$, the $\mathrm{EBV}^{+}$ type of GC has a better prognosis (12). This may depend on the immune response to EBV infection in humans. According to TCGA reports (5), programmed death 1 is frequently amplified in the $\mathrm{EBV}^{+}$type of $\mathrm{GC}$, suggesting the high immunogenicity of this type. In the present study, the MSI type accounted for $\sim 21.5 \%$ of all GC cases, was diagnosed at relatively older ages and was more common in females. Previous studies have shown that the majority of patients with MSI type exhibited intestinal-type GC predominantly located in the distal stomach; the majority of these patients were female and displayed an association with age $(5,13,14)$. In the present study, sequencing revealed that the mutation rate of $A R I D I A$ and $C D H 1$ were 21.5 and $3.1 \%$, respectively. According to previous TCGA reports (5), the GS type is mainly characterized by somatic gene mutations, including $C D H 1$ and ARIDIA. Unlike those TCGA reports, the mutation rate of $\mathrm{CDH} 1$ is lower in GS typing based on Chinese populations. Due to the limited number of mutations, the association between $\mathrm{CDH} 1$ mutations and prognosis had not been studied. However, Li et al (15) reported that patients with GC and $\mathrm{CDH} 1$ mutation had worse outcomes. According to the results of the present study, ARIDIA mutation showed poor prognosis, which was consistent with Ashizawa et al (16). Silencing the expression of the ARIDIA gene in GC cells could increase cell proliferation (17). These results suggested that the ARIDIA gene could inhibit the proliferation of cancer cells, thus acting as a tumor-suppressor gene. The clinical value of the ARIDIA gene should be further evaluated. CIN-subtype tumors accounted for $46.2 \%$ of 65 patients with GC, which occurred mainly at EGJ. This is similar to the results of Lim et al (18). In the present study, the abnormal amplification rate of EGFR in the CIN type of GC was $3.1 \%$, which was lower than that described in previous TCGA reports. EGFR plays a crucial role in the occurrence and development of GC. A previous study demonstrated that the EGFR gene was overexpressed in $32.7 \%$ of the samples and EGFR amplification occurred in $14.1 \%$ of the samples (19). The study also showed that $E G F R$ gene amplification was associated with the invasive ability of tumors (19). 
Its conclusions are basically the same as TCGA typing based on European populations in terms of proportion and clinical characteristics, but there are differences in gene amplification and gene mutation.

The MSI subtype has the best OS and lowest recurrence rate of all ACRG subtypes. Similar results were reported in the ACRG cohort (6). In the present study, low expression of CDH1 was defined as MSS/EMT type. The MSS/EMT type accounted for $20.0 \%$ of GC and its prognosis was the worst. These results are consistent with the clinical features of the MSS/EMT type in the ACRG cohort (6). Notably, MSS/EMT patients tended to be of diffuse type based on the Chinese population. Analysis of NGS and IHC revealed that there was no association between CDH1 and intestinal GC (20). This may be due to ethnic differences. In the present study, the mutation rate of TP53 was as high as $80.06 \%$ and TP53 mutation was an independent prognostic factor for GC. A number of studies have reported that TP53 is the most common mutant gene based on NGS and molecular profiling and contributes to the genesis and development of GC tumors $(5,6,20)$. The median OS of MSS/TP53 ${ }^{+}$was 29.5 months, which was slightly shorter than that of MSS/TP53-, with a median OS of 30.5 months. Similarly, the MSS/TP53+ type had a better outcome in the ACRG cohort vs. the MSS/TP53- type (6). Compared with TCGA typing, ARCG typing could better predict prognosis and was an independent prognostic factor for patients with GC. In the present study, the MSS/TP53- type was the most common (43.1\%), followed by the MSI type (21.5\%). In the ACRG cohort, the MSS/TP53- type was the most common subtype (43.75\%), followed by the MSS/TP53- type (28.13\%). The clinicopathological characteristics were not significantly correlated with the molecular typing of ACRG. In the ACRG cohort study, GC molecular subtype is significantly correlated with clinicopathological characteristics (6). These differences may be the result of a small sample size or individual differences. Recurrence is significantly associated with ACRG classification (21). Due to the lack of recurrence information, these analyses have not been performed in the present study although its results suggest that IHC can be used to replace NGS for ACRG typing of patients with GC. There were several differences in clinical characteristics and proportions between the present study and the ACRG cohort based on Japanese and Korean populations.

The number of cases collected in the present study is relatively small and the sample size needs to be expanded for further evaluation. Clinical case information needs to be further improved. In future studies, detailed stratified design based on EGFR amplification should be performed to evaluate the clinical efficacy of anti-EGFR gene drugs in patients with GC.

In conclusion, the molecular classification of ACRG can be classified by IHC and TCGA classification based on Chinese populations is basically the same as TCGA typing based on European populations in regard to proportion and clinical characteristics, but there are differences in gene amplification and gene mutation.

\section{Acknowledgements}

Not applicable.

\section{Funding}

The present study was supported by Hebei Science and Technology Subject Fund (grant no. 17397706D awarded to QZ) and the Excellent Clinial Medical Talents Training Program of Hebei Province (grant no.2019-139).

\section{Availability of data and materials}

TCGA datasets generated and/or analyzed during the current study are available in the NCBI database, https://www.ncbi.nlm. nih.gov/sra/PRJNA587325. The datasets used and/or analyzed during the current study are available from the corresponding author on reasonable request.

\section{Authors' contributions}

QW and QX performed the experiments. YL, HG, YR and JL analyzed the data. QW and QZ designed the experiments and drafted the manuscript. All authors read and approved the final manuscript.

\section{Ethics approval and consent to participate}

The present study was approved by the Medical Ethics Committee of The Fourth Hospital of Hebei Medical University and all patients provided informed consent.

\section{Patient consent for publication}

Not applicable.

\section{Competing interests}

The authors declare that they have no competing interests.

\section{References}

1. Bray F, Ferlay J, Soerjomataram I, Siegel RL, Torre LA and Jemal A: Global cancer statistics 2018: GLOBOCAN estimates of incidence and mortality worldwide for 36 cancers in 185 countries. CA Cancer J Clin 68: 394-424, 2018.

2. Chen W, Zheng R, Baade PD, Zhang S, Zeng H, Bray F, Jemal A, Yu XQ and He J: Cancer statistics in China, 2015. CA Cancer J Clin 66: 115-132, 2016.

3. Lee HS, Cho SB, Lee HE, Kim MA, Kim JH, Park DJ, Kim JH, Yang HK, Lee BL and Kim WH: Protein expression profiling and molecular classification of gastric cancer by the tissue array method. Clin Cancer Res 13: 4154-4163, 2007.

4. Shabani Azim F, Houri H, Ghalavand Z and Nikmanesh B: Next generation sequencing in clinical oncology: Applications, challenges and promises: A review article. Iran J Public Health 47: 1453-1457, 2018.

5. Cancer Genome Atlas Research Network: Comprehensive molecular characterization of gastric adenocarcinoma. Nature 513: 202-209, 2014.

6. Cristescu R, Lee J, Nebozhyn M, Kim KM, Ting JC, Wong SS, Liu J, Yue YG, Wang J, Yu K, et al: Molecular analysis of gastric cancer identifies subtypes associated with distinct clinical outcomes. Nat Med 21: 449-456, 2015.

7. Zou L, Wu Y, Ma K, Fan Y, Dong D, Geng N and Li E: Molecular classification of esophagogastric junction carcinoma correlated with prognosis. Onco Targets Ther 10: 4765-4772, 2017.

8. Robinson JT, Thorvaldsdóttir H, Wenger AM, Zehir A and Mesirov JP: Variant Review with the integrative genomics viewer. Cancer Res 77: e31-e34, 2017. 
9. Sinicrope FA, Ruan SB, Cleary KR, Stephens LC, Lee JJ and Levin B: bcl-2 and p53 oncoprotein expression during colorectal tumorigenesis. Cancer Res 55: 237-241, 1995

10. Thibodeau SN, French AJ, Roche PC, Cunningham JM, Tester DJ, Lindor NM, Moslein G, Baker SM, Liskay RM, Burgart LJ, et al: Altered expression of hMSH2 and hMLH1 in tumors with microsatellite instability and genetic alterations in mismatch repair genes. Cancer Res 56: 4836-4840, 1996.

11. Thibodeau SN, French AJ, Cunningham JM, Tester D, Burgart LJ, Roche PC, McDonnell SK, Schaid DJ, Vockley CW, Michels VV, et al: Microsatellite instability in colorectal cancer: Different mutator phenotypes and the principal involvement of hMLH1. Cancer Res 58: 1713-1718, 1998.

12. Kim SY, Park C, Kim HJ, Park J, Hwang J, Kim JI, Choi MG, Kim S, Kim KM and Kang MS: Deregulation of immune response genes in patients with Epstein-Barr virus-associated gastric cancer and outcomes. Gastroenterology 148: 137-147.e9, 2015.

13. Martinez-Ciarpaglini C, Fleitas-Kanonnikoff T, Gambardella V, Llorca M, Mongort C, Mengual R, Nieto G, Navarro L, Huerta M, Rosello S, et al: Assessing molecular subtypes of gastric cancer: Microsatellite unstable and Epstein-Barr virus subtypes. Methods for detection and clinical and pathological implications. ESMO Open 4: e000470, 2019.

14. Velho S, Fernandes MS, Leite M, Figueiredo C and Seruca R: Causes and consequences of microsatellite instability in gastric carcinogenesis. World J Gastroenterol 20: 16433-16442, 2014.

15. Li X, Wu WK, Xing R, Wong SH, Liu Y, Fang X, Zhang Y, Wang M, Wang J, Li L, et al: Distinct subtypes of gastric cancer defined by molecular characterization include novel mutational signatures with prognostic capability. Cancer Res 76: 1724-1732, 2016.
16. Ashizawa M, Saito M, Min AKT, Ujiie D, Saito K, Sato T, Kikuchi T, Okayama H, Fujita S, Endo H, et al: Prognostic role of ARID1A negative expression in gastric cancer. Sci Rep 9: 6769, 2019.

17. Hetmanski JHR, Schwartz JM and Caswell PT: Rationalizing Rac1 and Rho AGTPase signaling: A mathematical approach. Small GTPase 9: 224-229, 2018.

18. Lim B, Kim JH, Kim M and Kim SY: Genomic and epigenomic heterogeneity in molecular subtypes of gastric cancer. World J Gastroenterol 22: 1190-1201, 2016.

19. Birkman EM, Ålgars A, Lintunen M, Ristamäki R, Sundström J and Carpén O: EGFR gene amplification is relatively common and associates with outcome in intestinal adenocarcinoma of the stomach, gastro-oesophageal junction and distal oesophagus. BMC Cancer 16: 406, 2016.

20. Bria E, Pilotto S, Simbolo M, Fassan M, de Manzoni G, Carbognin L, Sperduti I, Brunelli M, Cataldo I, Tomezzoli A, et al: Comprehensive molecular portrait using next generation sequencing of resected intestinal-type gastric cancer patients dichotomized according to prognosis. Sci Rep 6: 22982, 2016.

21. Wang Q Liu G and Hu C: Molecular classification of gastric adenocarcinoma. Gastroenterology Res 12: 275-282, 2019.

(i) $($ ) This work is licensed under a Creative Commons Attribution-NonCommercial-NoDerivatives 4.0 International (CC BY-NC-ND 4.0) License. 